\title{
Partial Raphe Dysfunction in Neurotransmission Is Sufficient to Increase Mortality after Anoxic Exposures in Mice at a Critical Period in Postnatal Development
}

\author{
Karlene T. Barrett, ${ }^{1 *} \odot$ Ryan T. Dosumu-Johnson, ${ }^{2 *}$ J. Andrew Daubenspeck, ${ }^{1}$ Rachael D. Brust, ${ }^{2}$-Vasileios Kreouzis, ${ }^{2}$ \\ - Jun Chul Kim, ${ }^{3}$ Aihua Li, ${ }^{1}$ Susan M. Dymecki, ${ }^{2}$ and Eugene E. Nattie ${ }^{1}$ \\ 'Department of Physiology and Neurobiology, Geisel School of Medicine at Dartmouth, Lebanon, New Hampshire 03756, 2 Department of Genetics, Harvard \\ Medical School, Boston, Massachusetts 02115, and ${ }^{3}$ Department of Psychology, University of Toronto, Toronto, Ontario M5S, Canada
}

Sudden infant death syndrome (SIDS) cases often have abnormalities of the brainstem raphe serotonergic (5-HT) system. We hypothesize that raphe dysfunction contributes to a failure to autoresuscitate from multiple hypoxic events, leading to SIDS. We studied autoresuscitation in two transgenic mouse models in which exocytic neurotransmitter release was impaired via conditional expression of the light chain from tetanus toxin (tox) in raphe neurons expressing serotonergic bacterial artificial chromosome drivers Pet1 or Slc6a4. These used recombinase drivers targeted different portions of medullary raphe serotonergic, tryptophan hydroxylase $2(\mathrm{Tph} 2)^{+}$neurons by postnatal day (P) 5 through P12: approximately one-third in triple transgenic Pet1::Flpe, hßactin::cre, RC::PFtox mice; approximately three-fourths in Slc6a4::cre, RC::Ptox mice; with the first model capturing a near equal number of $P e t 1^{+}$,Tph2 ${ }^{+}$versus $\mathrm{Pet}^{+}, \mathrm{Tph} 22^{\text {low or negative }}$ raphe cells. At P5, P8, and P12, "silenced" mice and controls were exposed to five, $\sim 37$ s bouts of anoxia. Mortality was 5-10 times greater in "silenced" pups compared with controls at P5 and P8 $(p=0.001)$ but not P12, with cumulative survival not differing between experimental transgenic models. "Silenced" pups that eventually died took longer to initiate gasping $(p=0.0001)$, recover heart rate $(p=0.0001)$, and recover eupneic breathing $(p=0.011)$ during the initial anoxic challenges. Variability indices for baseline breathing distinguished "silenced" from controls but did not predict mortality. We conclude that dysfunction of even a portion of the raphe, as observed in many SIDS cases, can impair ability to autoresuscitate at critical periods in postnatal development and that baseline indices of breathing variability can identify mice at risk.

Key words: autoresuscitation; serotonergic system; sudden infant death syndrome

Significance Statement

Many sudden infant death syndrome (SIDS) cases exhibit a partial ( 26\%) brainstem serotonin deficiency. Using recombinase drivers, we targeted different fractions of serotonergic and raphe neurons in mice for tetanus toxin light chain expression, which prevented vesicular neurotransmitter release. In one model, approximately one-third of medullary Tph ${ }^{+}$neurons are silenced by postnatal (P) days 5 and 12, along with some Pet $1^{+}, \mathrm{Tph} 2^{\text {low or negative }}$ raphe cells; in the other, approximately three-fourths of medullary Tph $2^{+}$neurons, also with some Tph $2^{\text {low or negative }}$ cells. Both models demonstrated excessive mortality to anoxia (a postulated SIDS stressor) at P5 and P8. We demonstrated fatal vulnerability to anoxic stress at a specific time in postnatal life induced by a partial defect in raphe function. This models features of SIDS.

\section{Introduction}

Young mammals exposed to hypoxia-induced apnea and bradycardia can recover spontaneously. This process, called autoresus-

Received May 8, 2015; revised Feb. 20, 2016; accepted Feb. $26,2016$.

Author contributions: K.T.B., J.A.D., J.C.K., A.L., S.M.D., and E.E.N. designed research; K.T.B., R.T.D.-J., R.D.B., and V.K. performed research; R.D.B., J.C.K., and S.M.D. contributed unpublished reagents/analytic tools; K.T.B., R.T.D.-J., J.A.D., R.D.B., V.K., A.L., S.M.D., and E.E.N. analyzed data; K.T.B., R.T.D., S.M.D., and E.E.N. wrote the paper.

This work was supported by National Institutes of Health Program Project Grant HD036379 (Program Leader: S.M.D.; Project PIs: E.E.N. and S.M.D.), the (J Foundation for SIDS to K.T.B., National Institutes of Health General Medical Sciences Grant T32GM007753, Howard Hughes Medical Institute Gilliam Fellowship to R.T.D.-J., and Na- citation, is initiated by gasps, each associated with increased sympathetic activity, followed by cardiac and respiratory recovery (Stevens, 1965; Adolph, 1969; Guntheroth and Kawabori, 1975; Gershan et al., 1990, 1992; Melton et al., 1996; Fewell, 2005). Gasping presumably reoxygenates the brain to facilitate

tional Institutes of Health Grant F31 NS073276 to R.D.B. The content is solely the responsibility of the authors and does not necessarily represent the official views of the National Institute of General Medical Sciences or the National Institutes of Health. The authors thank Felicia L. Trachtenberg (New England Research Institutes, Watertown, Massachusetts) and Haiyi Xie (Community and Family Medicine, Geisel School of Medicine at Dartmouth, Lebanon, New Hampshire) for statistical assistance. 
cardiorespiratory recovery (St-John and Leiter, 2008), but it alone does not guarantee success in animals (Jacobi et al., 1991; Deshpande et al., 1999; Saiki et al., 2001) or infants (Meny et al., 1994; Poets et al., 1999; Sridhar et al., 2003).

Gasping is generated by neurons within the pre-Bötzinger complex (Lieske et al., 2000; Paton et al., 2006) whose discharge is influenced by serotonin (5-HT) (Peña and Ramirez, 2002; Harvey et al., 2006). Gasp initiation may occur without 5-HT, but 5-HT is essential to sustain gasping and restore breathing (Tryba et al., 2006; Toppin et al., 2007; St-John and Leiter, 2008; St-John et al., 2009) in autoresuscitation, independent of its effect on gasping. For example, in rodent pups with $80 \%-90 \%$ reduction in medullary 5-HT content (Erickson and Sposato, 2009; Cummings et al., 2011a,b), gasping is intact yet autoresuscitation is impaired and mortality increased.

Autoresuscitation failure may account for some cases of the sudden infant death syndrome (SIDS) (Guntheroth and Kawabori, 1975; Leiter and Böhm, 2007; Thach, 2008). Observations from SIDS infants being monitored indicate that gasping was present but was ineffective in restoring heart rate (HR) and breathing (Meny et al., 1994; Poets et al., 1999; Sridhar et al., 2003; Poets, 2004). Many SIDS cases demonstrate abnormalities in the medullary 5-HT system (Kinney et al., 2009), including a 26\% decrease in 5-HT (Duncan et al., 2010), a mild defect compared with published animal models of 5-HT deficiency and anoxic stress (Erickson and Sposato, 2009; Cummings et al., 2011a,b).

As a potential closer proxy to the moderate degree of brainstem 5-HT dysfunction in SIDS, we used two mouse models in which exocytic neurotransmission was disrupted (Kim et al., 2009) through the action of transgene-expressed light chain from tetanus toxin (tox) (Schiavo et al., 2000) in some, but not all, 5-HT neurons. In one model, triple transgenic Pet1::Flpe, hßact::cre, RC::PFTox mice (Kim et al., 2009), we estimate here that, at these early time points of P5 and P12, approximately one-third of medullary 5-HT neurons are targeted for tox expression and that there exist some Pet $1^{+}$neurons, which lack immunodetectable levels of tryptophan hydroxylase 2 (Tph2) protein. In the second model (double-transgenic Slc6a4::cre, RC::Ptox mice), we estimate approximately three-fourths of medullary 5-HT neurons are targeted, along with a modest fraction of raphe neurons, which lack immunodetectable levels of Tph2. In these two models, we test the hypothesis that raphe abnormalities perinatally and developmentally, even if limited to portions of the raphe population, can impair the ability to autoresuscitate; that successful autoresuscitation requires normal raphe neurotransmission. In these mice and their littermate controls, we measured baseline breathing, oxygen consumption, HR, and indices of breathing and HR variability (HRV), as well as the gasp latency, recovery of $\mathrm{HR}$ and breathing frequency, and survival after each of five, $37 \pm 4 \mathrm{~s}$ exposures to anoxia at P5, P8, and P12.

\section{Materials and Methods}

\section{Ethical approval}

All experimental protocols were approved by the Institutional Animal Care and Use Committee at Dartmouth College and at Harvard Medical School and were in accordance with the guidelines of the National Institutes of Health for animal care.

The authors declare no competing financial interests. *K.T.B. and R.T.D.-J. contributed equally to this work.

Correspondence should be addressed to Dr. Eugene E. Nattie, Department of Physiology and Neurobiology, Geisel School of Medicine at Dartmouth, Lebanon, NH 03756-0001. E-mail: Eugene.Nattie@Dartmouth.edu.

DOI:10.1523/JNEUROSCI.1796-15.2016

Copyright $\odot 2016$ the authors $\quad 0270-6474 / 16 / 363944-11 \$ 15.00 / 0$

\section{Experimental animals}

"Slc6a4::cre-silenced" pups were generated by crossing mice carrying RC::Ptox (Kim et al., 2009) to mice carrying the Slc6a4::cre transgene (Gong et al., 2007), whereas "Pet1::Flpe-silenced" pups were generated by crossing mice carrying RC::PFtox (Kim et al., 2009) with mice carrying both the Pet1::Flpe (Jensen et al., 2008) and $h \beta a c t:: c r e$ (Lewandoski et al., 1997) transgenes. In this paper we refer to these experimental or "silenced" mice as follows: Slc6a4::cre-silenced (Slc6a4::cre, RC::Ptox mice), and Pet1::Flpe-silenced (Pet1::Flpe, hßact::cre, RC::PFtox mice). We generated Slc6a4-Control mice (littermates of the Slc6a4:: cre-silenced pups) that carried the Slc6a4::cre transgene alone, the RC::Ptox allele alone, or no cre/tox transgene; and Pet1-Control mice (littermates of the Pet1::Flpe-silenced pups) that carried the RC::PFtox allele alone, Pet 1::Flpe, RC::PFtox (but no cre transgene), or $h \beta a c t:: c r e, R C::$ PFtox (but no Flpe transgene). Experiments were performed on a total of 116 Slc6a4::cre- and Pet1::Flpe-silenced and control pups at three postnatal ages (P5-P6, designated P5; P8-P9, designated P8; and P12-P13, designated $\mathrm{P} 12)$. The mice were housed in a temperature-controlled environment on a 12:12 h light-dark cycle in the Animal Resource Facility and had ad libitum access to standard rodent chow and water.

For experiments involving Slc6a4::cre-silenced mice, pups were obtained from 12 litters and consisted of 32 "silenced" (15 males, $17 \mathrm{fe}$ males) and 21 control pups ( 8 males, 13 females). For experiments involving Pet 1::Flpe-silenced mice, pups were obtained from 20 litters and consisted of 37 "silenced" ( 16 males, 21 females) and 26 control pups (15 males, 11 females).

\section{Genotyping}

The genotypes of Slc6a4::cre- and Pet1::Flpe-silenced and control pups were determined as previously described by Kim et al. (2009) and Ray et al. (2011). Briefly, DNA isolated from tail biopsies from P4 pups was subjected to PCR analysis using TaqDNA polymerase (New England BioLabs) and the following primer sequences (Invitrogen) that are complementary to DNA sequences encoding for Cre, Flpe, or tetanus toxin light chain (tox): 5'-GGCATGGTGCAAGTTGAATAACC-3' (cre forward primer); 5'-GGCTAAGTGCCTTCTCTACAC-3' (cre reverse primer); 5'-GCATCTGGGAGATCACTGAG-3' (Flpe forward primer); 5'-CCCATTCCA TGCGGGGTATCG-3' (Flpe reverse primer); 5' GCCGATCACCATCAACAACTTC-3' (tox forward primer); and $5^{\prime}$ GCAGAGCTTCACCAGCAACG-3' (tox reverse primer). The PCR protocol consisted of an initial denaturation at $94^{\circ} \mathrm{C}$ for $5 \mathrm{~min}$, followed by 35 cycles, each consisting of $94^{\circ} \mathrm{C}$ for $1 \mathrm{~min}, 60^{\circ} \mathrm{C}$ for $1.5 \mathrm{~min}$, and $72^{\circ} \mathrm{C}$ for $1 \mathrm{~min}$ and a final extension at $72^{\circ} \mathrm{C}$ for $10 \mathrm{~min}$. The PCR products generated were separated by agarose gel electrophoresis. The cre, Flpe, and tox amplification products separated on the agarose gel as 400, 800, and 200 bp PCR products, respectively. Slc6a4::cre-silenced pups were identified by the presence of both the cre and tox transgenes, whereas Pet1::Flpe-silenced pups were identified by the presence of all three transgenes, cre, Flpe, and tox, and the respective control pups lacked one or more of the transgenes.

\section{Experimental setup}

The experimental setup used in this study was similar to that described previously by Cummings et al. (2011a). Briefly, ventilation was measured using a head-out plethysmograph system consisting of a body chamber and a head chamber. The body chamber (volume $=\sim 60 \mathrm{ml}$; diameter $=$ $3 \mathrm{~cm}$, length $=8.5 \mathrm{~cm}$ ) was made from a water-jacketed glass cylinder with inlet and outlet ports that were connected to a water bath, allowing for continuous circulation of water around the chamber. The ambient temperature $\left(\mathrm{T}_{\mathrm{A}}\right)$ of the body chamber and thus the body temperature $\left(\mathrm{T}_{\mathrm{B}}\right)$ of the mouse pup were controlled by adjusting the temperature of the water circulating around the glass chamber. Both the $\mathrm{T}_{\mathrm{A}}$ and $\mathrm{T}_{\mathrm{B}}$ were continuously monitored with a thermistor probe and a fine thermocouple, respectively (Omega Engineering). The head chamber (volume $=$ $\sim 3 \mathrm{ml}$ ) was made from the bottom of a $50 \mathrm{ml}$ plastic syringe tube (Terumo Medical) with a piece of vinyl glove covering the larger of the two openings. A rubber gasket (Terumo Medical) was used to hold the piece of vinyl glove in place and to secure the head chamber into the anterior end of the body chamber. A small hole was made in the center of 
the vinyl glove, where the snout of the mouse pup was inserted and the hole was sealed with Impregum F polyether impression material (3M). The head chamber had an outlet port that was connected downstream to a pump (S-3A/I, AEI Technologies), which pulled air through the head chamber at a rate of $140 \mathrm{ml} / \mathrm{min}$. This high flow rate was chosen to prevent accumulation of $\mathrm{CO}_{2}$ in the head chamber and to ensure rapid delivery of the experimental gas to the animal. The air exiting the head chamber was passed through a Nafion drying tube (PerkinElmer) before being sampled by oxygen $\left(\mathrm{O}_{2}\right)$ and carbon dioxide $\left(\mathrm{CO}_{2}\right)$ analyzers (S-3A/I, AEI Technologies, and Capstar-100, CWE, respectively) to monitor oxygen consumption $\left(\dot{V}_{\mathrm{O}_{2}}\right)$. A pneumotach connected to the open end of the head chamber was attached to a differential pressure transducer (Validyne Engineering) to measure respiratory activity. Experimental gases were delivered to the head chamber via the open end of a $50 \mathrm{ml}$ syringe tube that was connected to the gas cylinder and then placed over the pneumotach. The pneumotach was calibrated by withdrawing and injecting $0.02 \mathrm{ml}$ of air into the head chamber, and the pressure signal associated with injection of this volume was integrated to determine the flow rate. HR was monitored with a telemetric device (CTA-F40, DSI), which consisted of 2 ECG leads that were placed on the surface of the pup's chest and held in place with a vest made from a cohesive flexible bandage (Andover).

\section{Methods}

The experimental protocol followed was modified from Cummings et al. (2011a). Breathing and HR were measured in Slc6a4::cre- and Pet1::Flpe-silenced and control pups at P5, P8, and P12 in room air (RA) and during 5 brief exposures to a gas mixture consisting of $97 \% \mathrm{~N}_{2} / 3 \%$ $\mathrm{CO}_{2}$ (anoxia). Each pup was separated from the litter, and the rectal temperature and body weight immediately recorded. Fur was removed from the area surrounding the snout and the surface of the chest. The pup was then fitted with the ECG telemetric device, and the snout inserted into the head chamber and sealed with the Impregum F polyether impression material. A fine thermocouple was inserted into the rectum for continuous monitoring of $\mathrm{T}_{B}$, and then the pup was placed in the body chamber where it was allowed to acclimatize in RA for $\sim 10$ min or until the pup was resting quietly and its $\mathrm{T}_{\mathrm{B}}$ was stabilized at $36 \pm 0.5^{\circ} \mathrm{C}$. The $\mathrm{T}_{\mathrm{A}}$ was adjusted to $35-36 \pm 0.5^{\circ} \mathrm{C}$ for $\mathrm{P} 5$ and $\mathrm{P} 8$ pups and $34-35 \pm 0.5^{\circ} \mathrm{C}$ for $\mathrm{P} 12$ pups to maintain the $\mathrm{T}_{\mathrm{B}}$ at $36 \pm 0.5^{\circ} \mathrm{C}$.

After acclimatization, the pup was allowed to breathe RA for $10 \mathrm{~min}$ to assess baseline cardiorespiratory parameters, followed by exposure to 5 brief bouts of anoxia separated by $\geq 5 \mathrm{~min}$ of RA, to assess the ability to autoresuscitate. Each anoxic exposure lasted $\sim 30-40 \mathrm{~s}$, and the pup was allowed to breathe RA for $5 \mathrm{~min}$ after the breathing frequency returned to normal before the subsequent anoxic exposure. In some instances, $>10$ min was required before the next anoxic exposure due to prolongation of the apnea. DataPac 2K2 data acquisition software system (RUN Technologies) was used to record the breathing and HR analog signals.

\section{Histology}

To assess cell specificity and coverage of recombinase activity from the Slc6a4::cre and Pet1::Flpe transgenes in medullary 5-HT neurons, we analyzed P5 and P12 double-transgenic Slc6a4::cre; Ai14 (Ai14 strain:

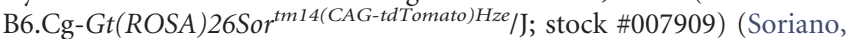
1999; Madisen et al., 2010) mice for tdTomato (tdT) expression, and double-transgenic Pet1::Flpe; RC::Fe (Brust et al., 2014) mice for GFP expression. Pups were anesthetized with 1.25\% Avertin (Sigma-Aldrich), perfused transcardially with ice-cold PBS followed by ice-cold 4\% PFA fixative solution, and brains removed and incubated overnight with gentle agitation in $4 \% \mathrm{PFA}$ at $4^{\circ} \mathrm{C}$. Subsequently, brains were incubated in a $30 \%$ sucrose solution (sucrose in $1 \mathrm{M}$ PBS) for $2 \mathrm{~d}$ at $4^{\circ} \mathrm{C}$, then frozen in tissue freezing medium (Triangle Biomedical Sciences) and cryosectioned coronally at $20 \mu \mathrm{m}$ thickness. Brain sections were collected directly onto superfrost-plus microscope slides (VWR International) and stored at $-80^{\circ} \mathrm{C}$ until processed.

Approximately every fifth brain section was processed for immunofluorescence detection of (1) Tph2, as an identifier of 5-HT neurons; and (2) the respective reporter molecule (tdT or GFP). Sections were incubated for $1 \mathrm{~h}$ at room temperature in blocking serum consisting of $5 \%$ normal donkey serum in PBS with 0.1\% Triton X-100 (PBS-T), followed by a $24-48 \mathrm{~h}$ incubation at $4{ }^{\circ} \mathrm{C}$ in primary antibody solution consisting of a 1:1000 (lot PL1986170) or 1:500 (lot PL201527) dilution of rabbit anti-Tph2 antibody (NB100-74555 Novus Biologicals). Included in the primary antibody incubation for double-transgenic Pet1::Flpe; RC::Fe tissue was 1:5000 dilution of chicken anti-GFP antibody (ab13970-100, Abcam). tdT expression in double-transgenic Slc6a4::cre; Ail4 tissue was detected via endogenous fluorescence. Following the $24-48 \mathrm{~h}$ incubation in primary antibody solution, sections were incubated in 1:500 dilutions of secondary antibody in PBS-T for $2 \mathrm{~h}$ at room temperature: Pet 1::Flpe; $R C:: F e$ tissue tissue was incubated in donkey anti-chick Alexa-488 and donkey anti-rabbit Cy3 (Jackson ImmunoResearch Laboratories, 703545-155 and 711-165-152, respectively); Slc6a4::cre; Ail4 tissue was incubated in donkey anti-rabbit Cy2 (Jackson ImmunoResearch Laboratories 711-225-152). In addition to the secondary antibody, a $5 \mu \mathrm{g} / \mathrm{ml}$ solution of DAPI in PBS was added to label cell nuclei. Recombinase negative sibling controls were stained with secondary antibody only, and then DAPI, to establish and assess background fluorescence. Slides were coverslipped and stored in the dark at $4^{\circ} \mathrm{C}$ until imaged. If tissue was missing or damaged during the staining process, a replacement (nearest adjacent) section was substituted.

To determine an index reflecting the relative number of $\mathrm{Tph} 2{ }^{+}$medullary cells, immunofluorescence-labeled brain sections were imaged using the $25 \times$ objective on a Zeiss LSM780 Laser Scanning Confocal Microscope (Carl Zeiss) using ZEN 2012 (ZEN version 2012, Carl Zeiss Imaging Solutions). Confocal stacks of approximately every fifth section throughout the medullary raphe and along the right lateral paragigantocellularis were collected, images imported into FIJI (http://fiji.sc/Fiji), and colocalization of reporter fluorescence (tdT or GFP) and Tph2 immunofluorescence in the $X, Y$, and $Z$ planes noted. DAPI signal was used to verify and aid in counting individual cells. Example photomicrographs were taken using a Zeiss Axioplan ( $10 \times$ objective) or a Zeiss LSM confocal $(25 \times$ and $40 \times$ objectives); tiled $z$ stacks were processed as maximum intensity projections in ImageJ. Photomicrographs were then background subtracted and had brightness adjusted for clarity. In three brains, each from independent litters, for each experimental group, we counted an average of 23 or 28 sections from the medullary raphe (rostral-most raphe magnus to caudal-most raphe obscurus) and an average of 22 or 26 sections for the lateral paragigantocellularis, for P5 and P12 tissue, respectively. Tph2-immunopositive neurons were counted and summed to yield a serotonergic neuron index. To determine the percentage of these medullary Tph2-immunopositive neurons that also had a history of recombinase activity and thus reporter or Tox expression, we tallied the colabeled neurons (reporter ${ }^{+}$and $\mathrm{Tph} 2^{+}$) and divided by the total number of $\mathrm{Tph} 2^{+}$neurons. Data are presented as mean $\pm \mathrm{SD}$. The effects of age and genotype on $\mathrm{Tph} 2{ }^{+}$cell number index and on the percentage of $\mathrm{Tph} 2{ }^{+}$cells also reporter positive were assessed by a two-way ANOVA using age and genotype as factors.

\section{Data analysis}

Mortality. The analysis of mortality resulting from anoxic exposures had two components. First, we examined cumulative survival in two initial cohorts. For Slc6a4::cre-silenced mice, an initial cohort of 26 "silenced" and 17 controls was studied at P5, P8, and P12; for Pet1::Flpe-silenced mice, in initial cohort of 19 "silenced" and 20 controls was studied at P5, P8, and P12. Cumulative survival of these cohorts is shown in Figure $2 C-E$. Second, to allow analysis of age dependence of survival we replaced pups that died at P5 or P8. At P12, the Slc6a4::cre-silenced "mixed" group included 12 pups previously exposed to hypoxia plus 2 pups exposed for the first time. The "mixed" control group included 18 pups previously exposed to hypoxia plus 1 pup exposed for the first time. At P12, the Pet1::Flpe-silenced "mixed" group included 4 pups previously exposed to hypoxia plus 9 pups exposed for the first time. The "mixed" control group included 16 pups that had been previously exposed to hypoxia and 2 being exposed for the first time.

Baseline breathing and HR. LabChart6 (AD Instruments), a digital strip chart recorder that also facilitates data calculations, was used to complete analysis of the data. The last minute of the breathing and HR tracings in RA before the first anoxic exposure was used to assess baseline 
breathing frequency $(f)$, tidal volume $\left(V_{T}\right)$, minute ventilation $\left(\dot{V}_{E} ; \mathrm{ml}\right.$ $\left.\min ^{-1} \mathrm{~g}^{-1}\right)$, oxygen consumption $\left(\dot{V}_{O_{2}} ; \mathrm{ml} \mathrm{min}^{-1} \mathrm{~g}^{-1}\right)$, and HR. We also normalized $\dot{V}_{E}$ and HR to $\dot{V}_{O_{2}}$. $\dot{V}_{E}$ was calculated as the product of the breathing frequency $\left(f ;\right.$ breaths $\left.\mathrm{min}^{-1}\right)$ and the tidal volume $\left(V_{T} ; \mathrm{ml}\right.$ $\left.\mathrm{g}^{-1}\right)$. The $f$ was obtained directly from the breathing tracings, whereas integration of the pressure changes associated with respiratory activity was used to calculate the $V_{T}$. $\dot{V}_{\mathrm{O}_{2}}$ was calculated as the product of the gas flow rate and the difference between the inspired and mixed expired $\mathrm{O}_{2}$ normalized to body weight $\left(\left[\right.\right.$ Flow $\left.(\mathrm{ml} / \mathrm{min}) \times\left(\mathrm{F}_{\mathrm{IO} 2}-\mathrm{F}_{\mathrm{EO} 2}\right)\right] /$ body weight $(\mathrm{g})$ ). HR was calculated directly from the ECG tracings.

Autoresuscitation. After each anoxic exposure, we measured the gasp latency (time to initiate gasping from the onset of primary apnea), the time to recover HR, and breathing frequency, to $90 \%$ of baseline.

Breathing and HRV. Three $1 \mathrm{~min}$ segments of continuous, nonoverlapping breathing and ECG tracings during the initial RA recordings were used to assess baseline variability in the interbreath intervals (IBIs; $\mathrm{s}^{-1}$ ) and the R-wave-to-R-wave intervals (RRI; $\mathrm{s}^{-1}$ ) in both the time and frequency domains. Custom-designed analysis software was used to assess linear and nonlinear measures of breathing and HRV. The SD, root of the mean square of successive differences (RMSSD) and an estimate of the area of the Poincare first return plot (major axis, $\mathrm{L} \times$ minor axis, $\mathrm{T}$; $\mathrm{L} \times \mathrm{T}$ ) was computed and used to evaluate the nature of interbeat and interbreath variability in the time domain (Carrasco et al., 2001). HRV in the frequency domain was estimated using the Lomb spectral analysis technique as proposed by Moody (1993) to avoid the spectral distortion caused by resampling of the original RRI time series. Spectral power between limits proposed by Just et al. (2000) were applied to the RRI power spectral density to estimate the low-frequency (LF) band power $(0.15-1.5 \mathrm{~Hz})$ and high-frequency $(\mathrm{HF})$ band power $(1.5-5.0 \mathrm{~Hz})$. The HF band is thought to reflect parasympathetic inputs to HR control, whereas the LF band is thought to reflect a combination of sympathetic and parasympathetic inputs to HR control (Chatow et al., 1995).

\section{Statistical analysis}

The data are presented as the mean \pm SD. The effects of gender and genotype on body weight (BW), body temperature, and baseline breathing and HR parameters were assessed by a two-way ANOVA at each age. When no gender differences were present, the data for both genders were combined and Student's $t$ test comparisons between genotypes were made at each age. Kaplan-Meier survival analyses were used to compare cumulative survival within each genotype compared with control for that genotype and survival at each age. To compare survival between the two experimental genotypes we applied a model with 2 factors: (1) experimental genotype (Pet1::Flpe-silenced vs Slc6a4::cre-silenced) and (2) tox expression (all "silenced," combining both experimental groups vs "nonsilenced control") and used Cox proportional hazard model to examine the effects of specific experimental genotype, tox expression in general, and their interaction, on survival. We also applied a Mantel-Cox log rank test with appropriate adjustment for multiple comparisons. The effects of gender and experimental genotype on the autoresuscitation parameters were assessed by a two-way ANOVA at each age. When no gender differences were present, the data for both genders were combined and reanalyzed by a one-way ANOVA at each age. Bonferroni post hoc comparisons were performed when significant effects were found. The effects of age and genotype on the indices of breathing and HRV were assessed by a two-way ANOVA followed by Bonferroni post hoc comparisons when significant interactions were found.

\section{Results}

\section{By P5 and P12, subsets, but not all medullary serotonergic neurons, are cumulatively captured by recombinase drivers}

To express tox, and thus suppress vesicular neurotransmission in 5-HT neurons, two different recombinase drivers, and thus two different engineered mouse models were used: Pet1::Flpe (Jensen et al., 2008) as the driver in the first mouse model and Slc6a4::cre (Gong et al., 2007) as the driver in the second. Both drivers use bacterial artificial chromosome (BAC) transgenics (Pet1 and Slc6a4 BACs, respectively) in which the transgene product (Flpe or Cre) is capable of recombining FRT- or lox $\mathrm{P}$-containing transgenes, respectively, in 5-HT neurons. To estimate the fraction of tox-manipulated 5-HT neurons achieved by these recombinase drivers by the ages of P 5 and P12 (as opposed to in the adult) (Gong et al., 2007; Jensen et al., 2008; Brust et al., 2014), we estimated the fraction of reporter-labeled 5-HT neurons (as a proxy for tox) in double-transgenic Pet1:: Flpe; $R C:: F e$ mice ( $R C:: F e$ is a ROSA26/CAG knock-in allele harboring a Flpe-dependent eGFP reporter) (Brust et al., 2014) and double-transgenic Slc6a4::cre; Ail4 (B6.Cg-Gt(ROSA) 26Sor ${ }^{\text {tm14(CAG-tdTomato } H z e}$ ) mice (the latter transgene is a ROSA26/ $C A G$ knock-in allele harboring a Cre-dependent red fluorescent tdT reporter) (Madisen et al., 2010) (Fig. 1). First, we determined an index reflective of the relative number of medullary serotonergic neurons in the P5 and P12 brainstem based on Tph2 immunopositivity; Tph2 is the rate-limiting enzyme in serotonin synthesis and thus one marker of serotonergic neuron identity. Cell counts were indistinguishable between genetic models, indicating neutrality of drivers and reporters with respect to serotonergic neuron populations, as would be expected by these neutral recombinase and reporter transgenes (Fig. $1 N$ ). For each model, three brains, each from independent litters, were analyzed. We then calculated for each model the percentage of Tph2-immunopositive neurons that were also reporter-positive, and thus captured by each driver. From this, we extrapolated an estimation of serotonergic neurons likely captured for tox expression and consequent neuronal silencing when these same drivers were used to recombine $R C:: P F t o x$ or its derivative $R C::$ Ptox (Kim et al., 2009), both knock-in alleles of the $R 26$ locus and thus likely similarly susceptible to recombination as the employed $R 26$ reporter alleles. We found that $\sim 35 \%$ (or onethird) of the medullary Tph2-immunopositive neurons expressed GFP in Pet1::Flpe; RC::Fe mice at P5 and P12 (Fig. 1O), with the greatest medullary colabeling observed in the rostral medulla in the area of the raphe magnus. As previously observed for Pet1 BAC drivers (Pelosi et al., 2014), we also observed that not all GFPmarked neurons expressed detectable levels of Tph2, suggesting that there exist a subset of raphe neurons that express the serotonergic transcription factor gene Pet1 but express low levels or no Tph2 at these time points. Grossly, this Pet $1^{+}, \mathrm{Tph} 2^{\text {negative or low }}$ subpopulation appeared comparable in magnitude to the double-positive cells within the medullary raphe, but varied along the rostrocaudal extent of the raphe. In all cases, GFP-marked cells were restricted to the brainstem raphe system and not found elsewhere in the brain. In the dorsal raphe system of this Pet1::Flpe model (Fig. 1D), the percentage Tph ${ }^{+}$cells captured was much greater, although not quantified here because of the substantive technical challenges around cell number and density. By contrast, in double-transgenic Slc6a4::cre; Ai14 mice (Fig. 1O), $\sim 73 \%$ (or three-fourths) of the medullary Tph2-immunopositive neurons expressed reporter, in this case tdTomato, thus capturing a majority portion of the 5-HT neuron population both at P5 and P12. Here too, not all tdTomato-marked raphe neurons expressed immunodetectable levels of Tph2, albeit at a lower frequency than in the Pet1::Flpe model. In this Slc6a4::cre model, double-positive cells $\left(\mathrm{Tph} 2^{+}, \mathrm{tdT}^{+}\right.$) distributed evenly across the medullary raphe, with only a small subset of $\mathrm{tdT}^{+}$, Tph $2^{\text {negative or low }}$ raphe cells. Of note, Slc6a4::cre; Ai14 mice too showed reporter expression in nonserotonergic cells outside the raphe system, including the thalamus, cerebral cortex, and cerebellum. Within the medullary raphe, though, very few reporter-positive, Tph $2^{\text {negative or low }}$ neurons were observed. In the medulla, these two drivers (Pet1::Flpe and Slc6a4::cre) overlapped in neurons captured most substantially in the raphe magnus. 
A

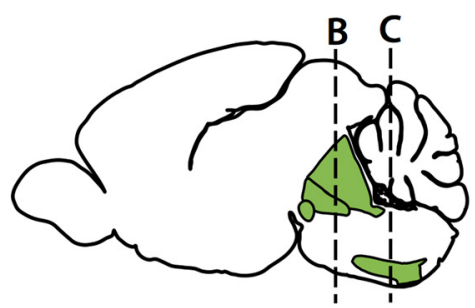

B

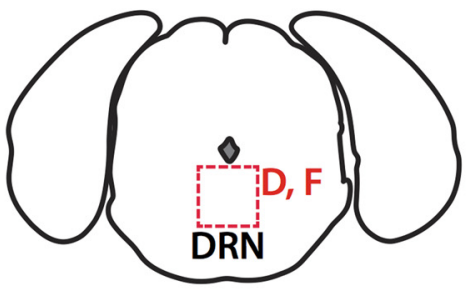

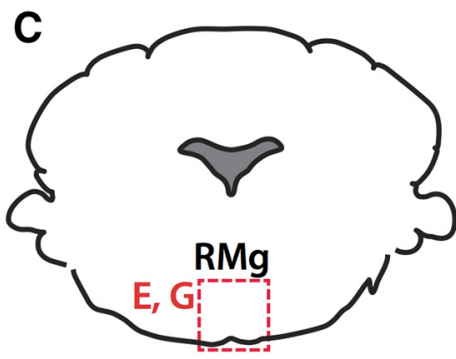

\section{P12 mouse brain}
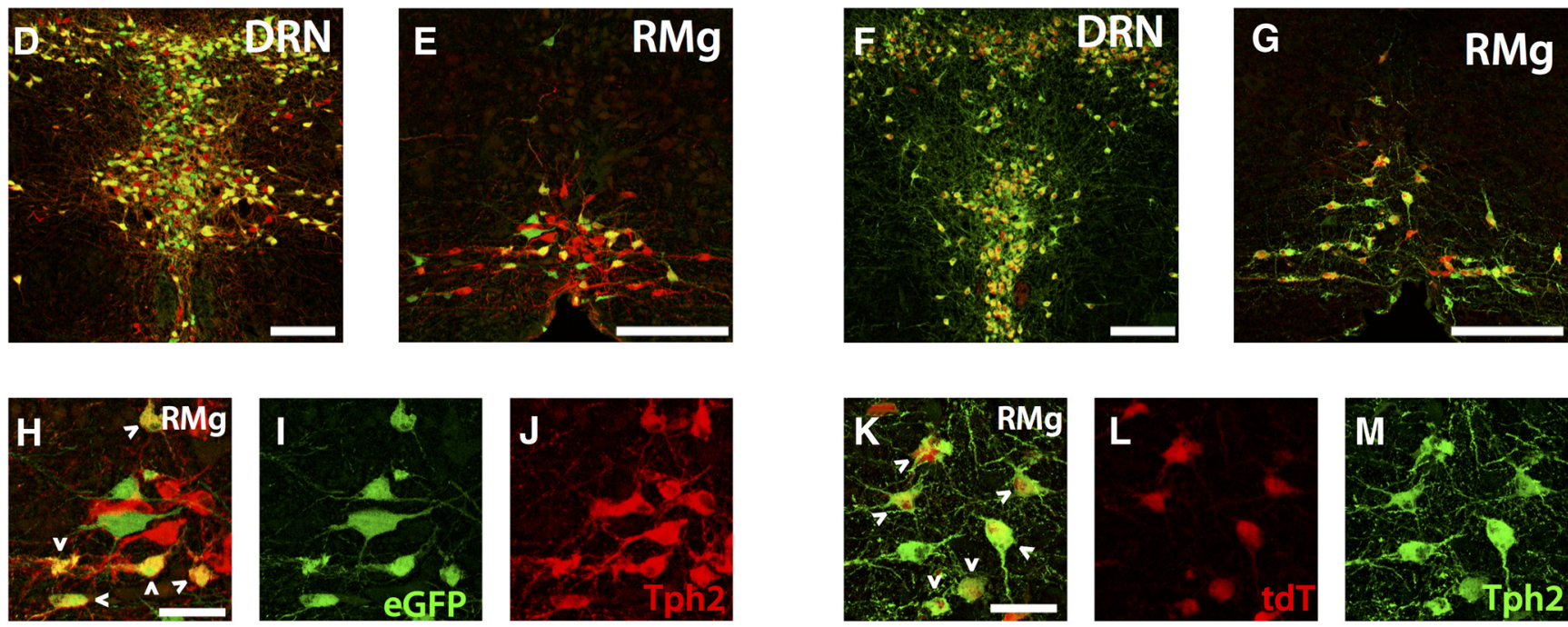

\section{Pet1::Flpe; RC::Fe}
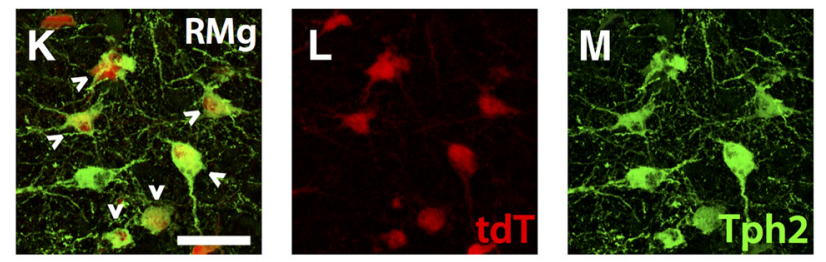

\section{Slc6a4::cre; Ai14}
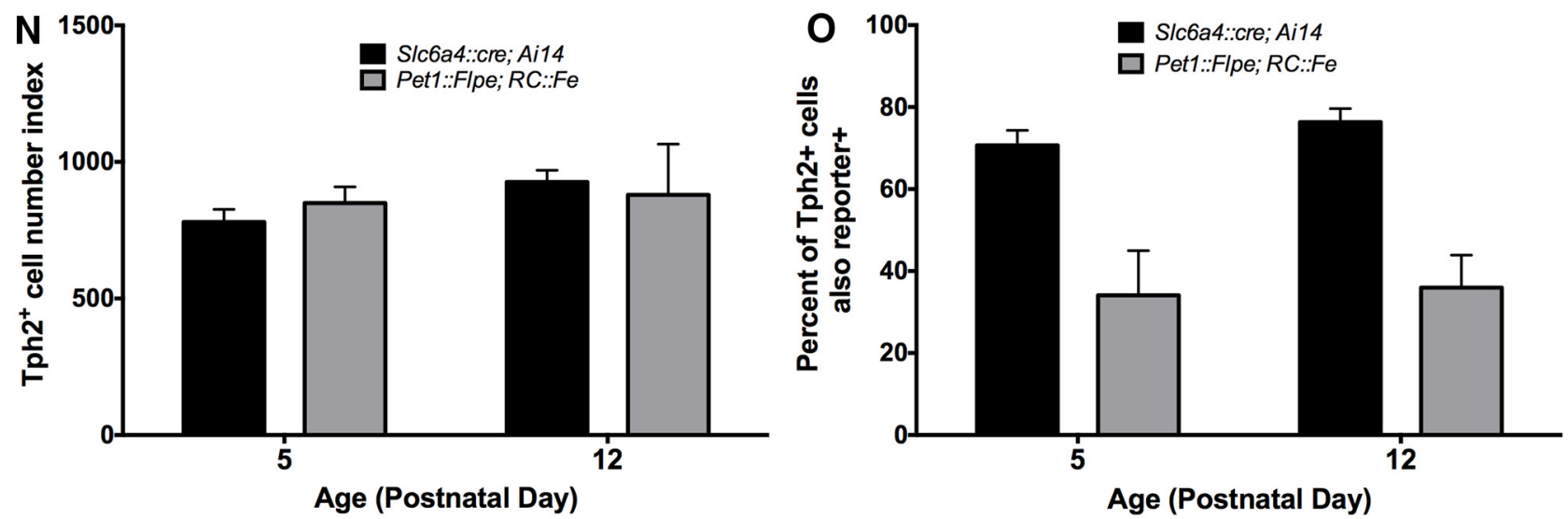

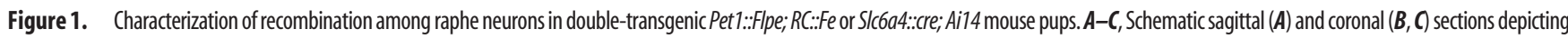
the dorsal raphe nucleus (DRN) and medullary raphe (raphe magnus [RMg]) regions presented in $\mathbf{D}-\mathbf{G}$. $\mathbf{D}-\mathbf{M}$, Representative images of $20 \mu \mathrm{m}$ coronal brain sections from the Pet1::Flpe model (doubletransgenic Pet1:.:Flpe; $R C$ (.:Fe pups) at P12 showing the DRN (D) and RMg (E, $\boldsymbol{H}-\boldsymbol{J})$ in which Pet1.:Flpe-captured neurons express eGFP from the conditional $R 26$ reporter $R(: .: F e$, detected by indirect immunofluorescence in green (Alexa-488), whereas Tph2 immunopositivity was detected in red (Cy3). Double-labeled cells ( ${ }^{\wedge}$ ) arehighlighted in the composite $\boldsymbol{H}$, versus single-channel fluorescence images of $\mathbf{I}$ and $J . \boldsymbol{K}-\boldsymbol{M}$, Representative images of $20 \mu \mathrm{m}$ coronal brain sections from the Slc6a4.:cre model (double-transgenic Slc6a4:.cre; Ai14 pups) at P12 showing the DRN $(\boldsymbol{F})$ and RMg $(\boldsymbol{G}, \boldsymbol{K}-\boldsymbol{M})$ in which Slc6a4:.cre-captured neurons express $\mathrm{tdT}$ (red) from the conditional R26 reporter Ai74, detected by direct fluorescence, whereas Tph2 immunopositivity was detected in green (Cy2). Double-labeled cells ( $\left.{ }^{(}\right)$are highlighted in the composite $\boldsymbol{K}$, versus single-channel fluorescence images of $\boldsymbol{L}$ and $\boldsymbol{M} . \boldsymbol{N}$, Medullary Tph $2^{+}$cell counts (mean \pm SD) at P5 and P12 (3 animals per genotype and age, each from an independent litter). Two-way ANOVA with genotype and age as factors revealed no significant effects, $p=0.85$. O, Percentage of Tph2-immunopositive neurons also reporter-positive (mean \pm SD) at P5 and P12 ( 3 animals per genotype and age, each from an independent litter) showed significant effects of genotype both at $\mathrm{P5}(p<0.001)$ and $\mathrm{P} 12(p<0.001)$, based on two-way ANOVA with age and genotype as factors followed by post hoc comparisons (Bonferonni). Scale bars: $\mathbf{D}-\mathbf{G}, 200 \mu \mathrm{m} ; \boldsymbol{H}, \boldsymbol{K}, 50 \mu \mathrm{m}$. 
Table 1. Baseline characteristics of SIc6a4::cre-silenced and control pups and Pet1::Flpe-silenced and control pups studied at P5, P8, and P12 ${ }^{a}$

\begin{tabular}{|c|c|c|c|c|c|c|c|c|}
\hline Age & Genotype & $n$ & $\begin{array}{l}\text { Body } \\
\text { weight (g) }\end{array}$ & $\begin{array}{l}\text { Breathing } \\
\text { frequency } \\
(f ; \text { min })\end{array}$ & $\begin{array}{l}\text { Tidal volume } \\
\left(\mathrm{V}_{\mathrm{T}} ; \mathrm{ml} / \mathrm{kg}\right)\end{array}$ & $\begin{array}{l}\text { Minute ventilation } \\
\left(\dot{V}_{E} \mathrm{ml} \cdot \min ^{-1} \cdot \mathrm{kg}^{-1}\right)\end{array}$ & $\begin{array}{l}\text { Metabolic rate } \\
\left(\dot{V}_{\mathrm{O}_{2}} \mathrm{ml} \cdot \mathrm{min}^{-1} \cdot \mathrm{kg}^{-1}\right)\end{array}$ & $\mathrm{HR}$ (bpm) \\
\hline P5 & Control & 17 & $3.7(0.7)$ & $221(26)$ & $5.5(0.81)$ & $1,110(100)$ & $52.0(8.6)$ & $585(44)$ \\
\hline Slc6a4::cre-silenced & & 26 & $3.3^{*}(0.6)$ & $199 *(33)$ & $5.6(0.97)$ & $1,080(140)$ & $49.0(11)$ & $564(49)$ \\
\hline P8 & Control & 19 & $5.2(0.7)$ & $251(26)$ & $4.5(0.53)$ & $1,130(120)$ & $48.0(11)$ & $598(41)$ \\
\hline Slc6a4::cre-silenced & & 23 & $4.1^{* * *}(0.7)$ & $210^{* * *}(27)$ & $5.3^{* * *}(0.60)$ & $1,120(110)$ & $45.0(5.5)$ & $552^{* *}(32)$ \\
\hline P12 & Control & 19 & $7.3(1.2)$ & $226(30)$ & $5.2(0.88)$ & $1,160(230)$ & $51.0(6.3)$ & 609 (37) \\
\hline Slc6a4::cre-silenced & & 14 & $5.6^{* *}(1.4)$ & $198^{*}(39)$ & $6.3^{*}(1.3)$ & $1,200(190)$ & $50.0(6.6)$ & $613(40)$ \\
\hline P5 & Control & 20 & $4.0(0.7)$ & $218(40)$ & $5.2(1.3)$ & $1,100(190)$ & $50.0(5.5)$ & $619(39)$ \\
\hline Pet1::Flpe-silenced & & 19 & $3.4^{*}(0.6)$ & $188^{*}(39)$ & $6.0(1.6)$ & $1,070(130)$ & $45.0^{*}(6.7)$ & $554^{* * *}(41)$ \\
\hline P8 & Control & 20 & $5.7(0.9)$ & $249(20)$ & $4.4(0.5)$ & $1,100(110)$ & $49.0(5.4)$ & $636(45)$ \\
\hline Pet1::Flpe-silenced & & 16 & $4.5^{* * *}(0.5)$ & $217^{* *}(32)$ & $5.2^{* * *}(0.9)$ & $1,120(150)$ & $49.0(5.0)$ & $597^{*}(46)$ \\
\hline P12 & Control & 18 & $8.9(1.8)$ & $235(29)$ & $4.4(0.73)$ & $1,020(160)$ & $50.0(8.9)$ & $662(39)$ \\
\hline Pet1::Flpe-silenced & & 13 & $7.8^{* *}(1.9)$ & $225(30)$ & $5.9^{* *}(1.2)$ & $1,310(290)$ & $58.0^{*}(13)$ & 656 (49) \\
\hline
\end{tabular}

${ }^{a}$ Data are mean \pm SD. The ambient temperature was $35-36 \pm 0.5^{\circ} \mathrm{C}$ at $\mathrm{P} 5$ and $\mathrm{P} 8$ and $34-35 \pm 0.5^{\circ} \mathrm{C}$ at $\mathrm{P} 12$.

${ }^{*} p<0.05 ;{ }^{* *} p<0.001 ;{ }^{* * *} p<0.0001$; significant differences from controls (Student's $t$ test comparing genotypes at each age).

\section{Baseline physiological characteristics}

Slc6a4::cre-silenced pups were significantly smaller than control pups at all ages by 11\%-23\% (P5: $p=0.028, \mathrm{P} 8: p=0.0001, \mathrm{P} 12$ : $p=0.001)$. They had significantly slower breathing than controls at all ages by $9 \%-16 \%$ (P5: $p=0.023$, P8: $p=0.0001, \mathrm{P} 12: p=$ $0.024)$, but their $V_{T}$ was significantly larger at $\mathrm{P} 8$ and $\mathrm{P} 12$ by $18 \%-21 \%(\mathrm{P} 5: p=0.134, \mathrm{P} 8: p=0.0001, \mathrm{P} 12: p=0.009)$. There was no significant difference in $\dot{V}_{E}$ (P5: $p=0.463$, P8: $p=0.821$, P12: $p=0.665)$ or $\dot{V}_{\mathrm{O}_{2}}(\mathrm{P} 5: p=0.314, \mathrm{P} 8: p=0.243, \mathrm{P} 12: p=$ $0.661)$ at any age. HR was slightly $(46 \mathrm{bpm})$ but significantly slower than control pups only at P8 (P5: $p=0.158$, P8: $p=$ 0.0002, P12: $p=0.763$ ) (Table 1).

Pet1::Flpe-silenced pups were also significantly smaller than control pups at P5 and P8 by 15\%-21\% (P5: $p=0.011$, P8: $p<$ $0.0001, \mathrm{P} 12: p=0.092)$. They too had significantly slower breathing than control pups at $\mathrm{P} 5$ and $\mathrm{P} 8$ by $14 \%(\mathrm{P} 5: p=0.024, \mathrm{P} 8: p=$ $0.0008, \mathrm{P} 12: p=0.374)$, and their $V_{T}$ was significantly larger at $\mathrm{P} 8$ and $\mathrm{P} 12$ by $18 \%-34 \%$ (P5: $p=0.109, \mathrm{P} 8: p=0.0008, \mathrm{P} 12: p=$ 0.0002 ), which in this case resulted in a $28 \%$ larger $\dot{V}_{E}$ at P12 (P5: $p=0.573$, P8: $p=0.621, \mathrm{P} 12: p=0.001) . \dot{V}_{\mathrm{O}_{2}}$ was slightly but significantly lower at P5 and higher at P12 (P5: $p=0.018, \mathrm{P} 8: p=$ $0.988, \mathrm{P} 12: p=0.049)$. HR was significantly slower at P5 and P8 by 6\%-10\% (P5: $p<0.0001$, P8: $p=0.015$, P12: $p=0.684$ ). There were no gender differences in baseline characteristics in either of the two mouse models. These changes in body weight, $f$, and $V_{T}$ are similar to findings in other, more severe models of 5-HT dysfunction (Erickson and Sposato, 2009; Cummings et al., 2011a,b).

\section{Failed autoresuscitation in Slc6a4::cre-silenced and Pet1::Flpe-silenced pups at a critical age}

Results from study of the initial cohort subjected to repeated anoxia at $P 5, P 8$, and $P 12$

In both Slc6a4::cre-silenced and Pet1::Flpe-silenced pups, autoresuscitation was less effective compared with controls following repeated episodic exposure to anoxia and resulted in increased mortality compared with control pups at P5 and P8. Typical examples of HR and breathing responses during successful and failed autoresuscitation are shown in Figure 2, $A$ and $B$, respectively. In general, successful autoresuscitation was characterized by a brief primary apnea and rapid recovery of normal HR and eupneic breathing following the onset of gasping (Fig. 2A), whereas failed autoresuscitation was characterized by a prolonged primary apnea (delayed gasping) and an inability to recover $\mathrm{HR}$ and eupneic breathing despite vigorous gasping (Fig. 2B).

Of the 26 Slc6a4::cre-silenced pups tested initially at P5, only $42 \%$ (11 of 26) survived to the endpoint of the study at P12 (cumulative survival), compared with 94\% (16 of 17) survival among the control pups (Fig. $2 C ; p=0.001$ ). All deaths occurred during the anoxic challenge at P5-P6 or P8-P9, except for one control and one "silenced" pup that died of unknown causes in their home cages between test days.

Similarly, of the 19 Pet1::Flpe-silenced pups tested initially at P5, only 16\% (3 of 19) survived to the endpoint of the study at P12 (cumulative survival), compared with 75\% (15 of 20) survival among the control pups (Fig. $2 D ; p=0.001$ ). All deaths occurred during the anoxic challenge on experimental days P5-P6 and P8, except for one "silenced" and three control pups that died of unknown causes in their home cages between test days.

We compared the cumulative survival between Slc6a4:: cre-silenced and Pet1::Flpe-silenced pups (shown in Fig. 2) by applying Cox proportional hazard model to examine the effects of experimental genotype (Pet1::Flpe-silenced vs Slc6a4:: cre-silenced), tox expression (all silenced, expressing tox, vs all control, not expressing tox), and their interaction on survival time. We apply statistically the word "genotype" to refer to (1) Slc6a4::cre-silenced and (2) Pet1::Flpe-silenced (i.e., each of these two groups is a separate genotype). As there can be tox expression in both of these genotypes, we also apply statistically whether or not there is tox expression as a parameter to be analyzed. The test was designed to ask whether there is a significant difference in survival between Slc6a4::cre-silenced and Pet1::Flpe-silenced pups (i.e., between the two genotypes). This distinction is necessary as one initial hypothesis was that Slc6a4::cre-silenced might differ from Pet $1:$ Flpe-silenced in survival given the different fraction of manipulated serotonergic cells.

The main effects of both genotype (Slc6a4::cre-silenced vs Pet 1::Flpe-silenced) and tox expression (all silenced vs all control) were significant ( $p=0.0460$ and $p=0.0002$; respectively), but the interaction between them was not $(p=0.5431)$. We also applied a Mantel-Cox log rank test with appropriate adjustment for multiple comparisons. Survival differences between each control and experimental genotype were highly significant $(p<$ $0.0001)$; survival differences between the two models of toxexpressing mice were not significant $(p=0.3299)$. There was no 
A Successful autoresuscitation in a P8 control pup

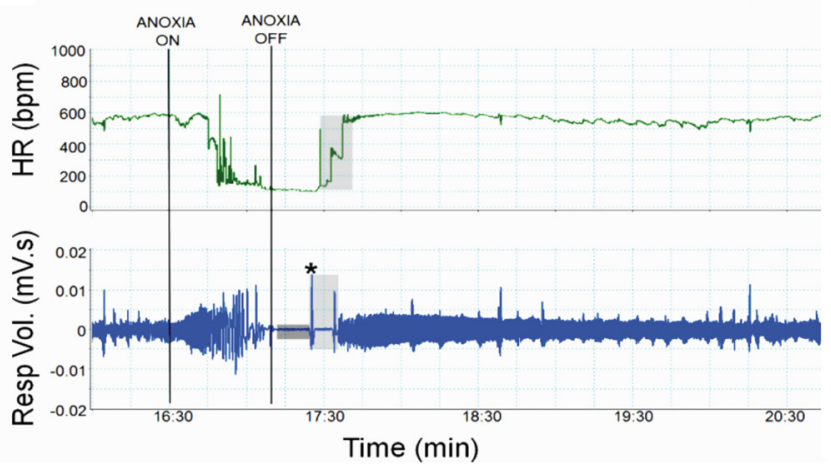

C

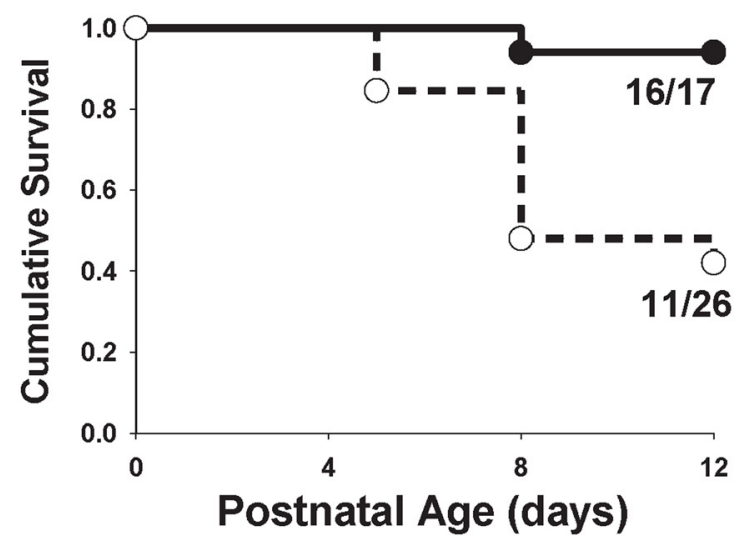

B Failed autoresuscitation in a P8 Pet1::Flpe-silenced pup

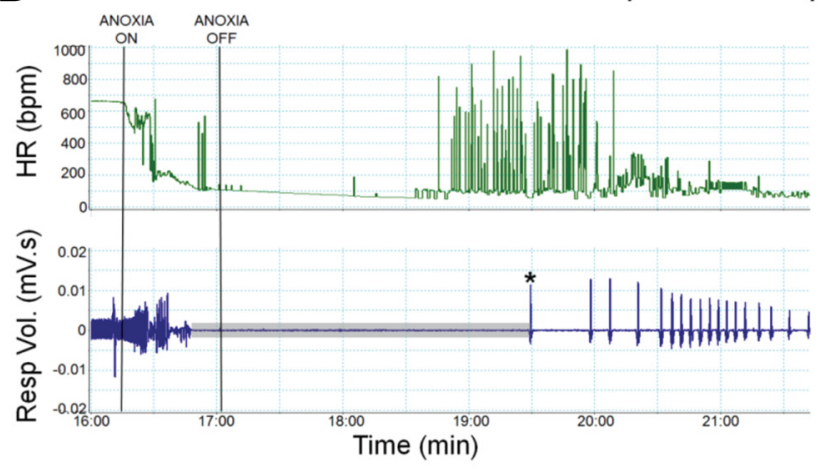

D Pet1::Flpe

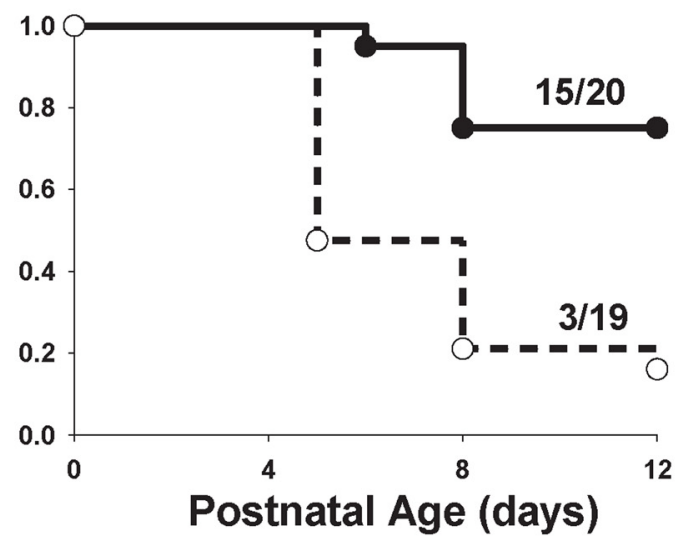

$\mathbf{E}$
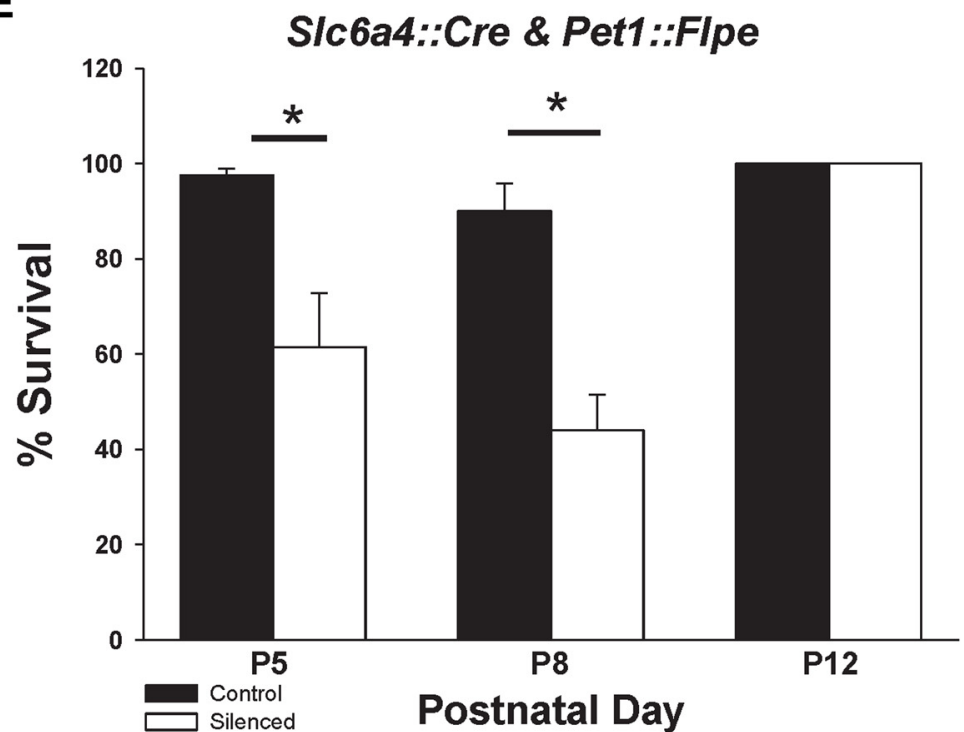

Figure 2. Increased mortality following repeated anoxic exposure in S/c6a4::cre- and Pet 1::Flpe-silenced pups at a critical period in postnatal development. Typical examples of HR (top) and breathing (bottom) responses to anoxia in a P8 control pup during successful autoresuscitation $(\boldsymbol{A})$ and in a P8 Pet 1 ::Flpe-silenced pup during failed autoresuscitation (B). Resp Vol., Respiratory volume here expressed simply as the raw tracing in $\mathrm{mV} / \mathrm{s}$ as for the purposes of this study quantification is not required. $\boldsymbol{C}, \boldsymbol{D}$, Kaplan-Meier cumulative survival analysis for initial cohorts of (C) S/c6a4:: cre-silenced $(n=26)$ and control $(n=17)$ pups and (D) Pet 1::Flpe-silenced $(n=19)$ and control $(n=20)$ pups during the anoxic challenge at P5, P8, and P12 (for statistics, see text). $E$, Combined survival results of both S/c6a4::cre-silenced and Pet 1::Flpe-silenced and controls in the initial cohort. At both P5 and P8, there is significantly less survival in silenced pups. ${ }^{*} p=0.01$ (ANOVA at each age, with survival and genotype, silenced vs nonsilenced, as factors). At P12, there was no effect on survival of 5 -HT neuron silencing. 
significant difference in survival time between control pups and tox-expressing pups that depended on experimental genotype (i.e., the difference in survival between tox-expressing and non-tox-expressing pups was statistically the same between Slc6a4::cre- and Pet1::Flpe-silenced pups). There were no gender differences in the number of Slc6a4::cre- or Pet1::Flpe-silenced and control pups that failed to autoresuscitate at any age.

Given that there was no significant difference in survival between the two experimental groups (Pet1::Flpe and Slc6a4:: cre silenced), we combined the results at the different ages to emphasize the age dependence of survival (Fig. 2E). Although overall at both P5 and P8 there is significantly less survival in silenced pups, by P12 there was no effect on survival of 5-HT neuron silencing within the cohort of pups shown in Figure 2.

Results from study of repeated anoxia at P5, P8, and P12, including pups added to replace those that succumbed (data not shown)

To further examine whether there was an age dependence in the ability to survive repeated bouts of anoxia, we added pups after P5 to replace pups that died. There remains no effect of repeated anoxia on survival at P12 (data not shown). At P12, the Slc6a4::cre-silenced group included 12 pups previously exposed to anoxia plus 2 pups exposed for the first time; the controls included 18 pups previously exposed to anoxia plus 1 pup exposed for the first time. The Pet1::Flpe-silenced group included 4 pups previously exposed to anoxia plus 9 pups exposed for the first time; the controls included 16 pups that had been previously exposed to anoxia and 2 being exposed for the first time. Thus, the high survival of silenced pups at P12 was not clearly related to the presence or absence of prior anoxic exposure.

\section{Gasping, HR, and eupnea recovery in autoresuscitation}

Slc6a4::cre-silenced pups that went on to die at P5 showed abnormal responses to the anoxic challenges in the assay compared with sibling control pups: they took $\sim 3$ times longer to initiate gasping (Fig. $3 A ; p=0.0001$ ), $\sim 5$ times longer to recover $\mathrm{HR}$ to $90 \%$ of baseline (Fig. $3 A ; p=0.0001$ ), and $\sim 2$ times longer to recover eupneic breathing to $90 \%$ of baseline (Fig. $3 A ; p=0.011$ ). Compared with Slc6a4::cre-silenced pups that survived, Slc6a4::cre-silenced pups that went on to die at P5 took $\sim 2$ times as long to initiate gasping $(p=0.0002)$ and $\sim 2$ times as long to recover normal HR to $90 \%$ of baseline $(p=0.0009)$. Compared with controls, Slc6a4::cre-silenced pups that survived had an $\sim 2$ fold longer time to initiate gasping (Fig. $3 A ; p=0.0005$ ), but recovery times of $\mathrm{HR}$ and eupnea were unaffected.

Similarly, Pet1::Flpe-silenced pups that ultimately died at P5 compared with controls took $\sim 4$ times longer to initiate gasping (Fig. $3 B ; p=0.0001$ ) and $\sim 4$ times longer to recover $\mathrm{HR}$ and eupneic breathing to $90 \%$ of baseline (Fig. $3 B ; p=0.0004$ and $p=$ 0.0002, respectively). Compared with Pet1::Flpe-silenced pups that survived, Pet $1:$ Flpe-silenced pups that died also took $\sim 4$ times longer to initiate gasping $(p=0.0001)$ and to recover eupneic breathing $(p=0.0056)$. The times to initiate gasping and to recover $\mathrm{HR}$ and eupneic breathing to $90 \%$ of baseline in Pet1::Flpe-silenced pups that survived were not significantly different from controls that survived.

Inspection of Figure 3 suggests that, in both the Slc6a4::creand Pet1::Flpe-silenced and control pups, the times to recover normal HR and eupneic breathing were directly associated with the gasp latency. Linear regression analysis using the individual data points summarized in Figure 3 shows that, in Slc6a4::cre-silenced pups: HR and eupneic recovery times versus
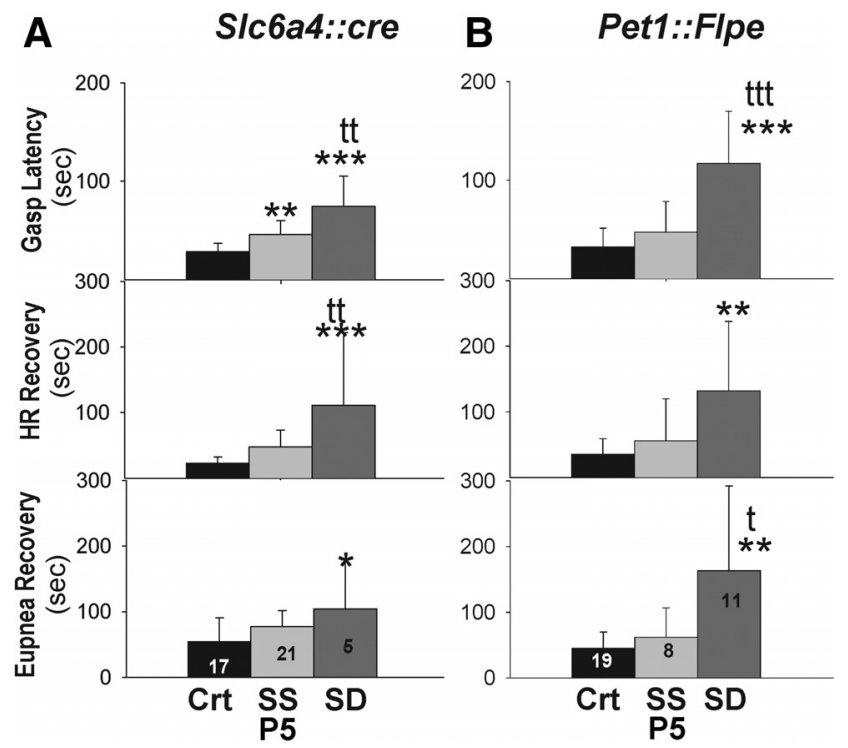

Figure 3. Prolonged gasp latency and delayed recovery of HR and eupnea following anoxic exposure among SIc6a4::Cre- and Pet1::Flpe-silenced pups at postnatal (P) day 5. A, SIc6a4::cre pups at P5: $n=17$ for control pups (Crt); $n=21$ for "silenced" pups that survived (SS); $n=5$ for "silenced" pups that died (SD). B, Pet1::Flpe pups at P5: $n=19$ for control pups (Crt); $n=8$ for "silenced" pups that survived (SS); $n=11$ for "silenced" pups that died (SD). * $p=0.01$, significant differences from controls that survived. ${ }^{* *} p=0.001$, significant differences from controls that survived. ${ }^{* *} p=0.0001$, significant differences from controls that survived. ${ }^{\dagger} p=0.01$, significant differences from "silenced" pups that survived. ${ }^{+\dagger} p=0.001$, significant differences from "silenced" pups that survived. ${ }^{+t \dagger} p=0.0001$, significant differences from "silenced" pups that survived. The data are presented as the mean \pm SD. One-way ANOVA at each age, with genotype as the between-subjects factor; Bonferroni post hoc comparisons.

gasp latency are significantly related $\left(p=0.001, R^{2}=0.68\right.$ and $p=0.001, R^{2}=0.3$, respectively); and in Pet $1:$ Flpe-silenced mice: HR and eupneic recovery times versus gasp latency are significantly related ( $p=0.001, R^{2}=0.68 ; p=0.001, R^{2}=0.63$, respectively).

\section{Can variability of baseline breathing and HR in Slc6a4::cre- and Pet1::Flpe-silenced pups predict autoresuscitation failure?}

We evaluated whether variability of $\mathrm{HR}$ or breathing measured at P5 would predict subsequent mortality. With respect to HRV measured as SD, RMSSD, major axis of Poincare first return plot $(\mathrm{L}) \times$ minor axis of Poincare first return plot $(\mathrm{T}), \mathrm{LF}$, or HF of the RR interval, there was no significant effect of genotype at P5 in either Slc6a4::cre-silenced or Pet1::Flpe-silenced pups compared with control pups, nor was there a significant effect of gender (data not shown).

Breathing variability measured at P5 as SD, RMSSD, and L $\times$ $\mathrm{T}$ of the IBI differed by genotype (Fig. 4). At P5, there was a significant effect of genotype in Pet1::Flpe-silenced pups with a greater SD $(p=0.041)$ and RMSSD $(p=0.025)$ and in Slc6a4::cre silenced pups with a greater SD $(p=0.00982), \operatorname{LxT}(p=0.0051)$, and RMSSD ( $p=0.00666)$. There was no effect of gender. To test for whether the significant genotype effect could predict mortality, we applied the Bonferroni post hoc test comparing silenced pups that died, or survived, to controls. At P5 in Slc6a4::cre-silenced pups compared with controls, the SD and the RMSSD of the IBI were greater in those that died ( $p=$ 0.0099 and $p=0.0073$, respectively) and in those that survived ( $p=0.0147$ and $p=0.0071$, respectively). $\mathrm{L} \times \mathrm{T}$ was greater only in silenced pups that died $(p=0.0026)$. At P5 in 


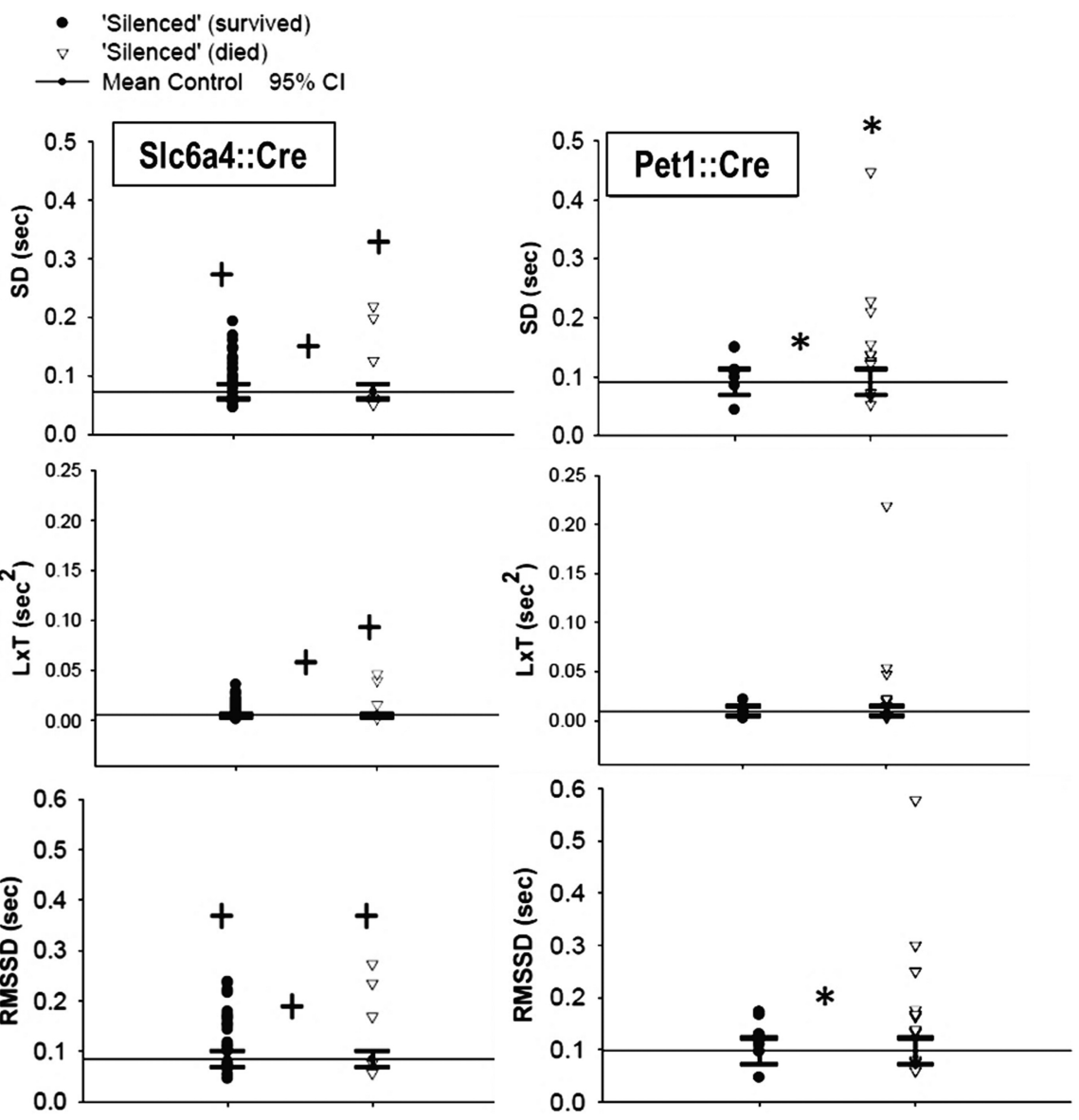

Figure 4. Variability for the baseline RA IBI in SIc6a4::"cre- and Pet1::Flpe-silenced and control pups studied at P5 expressed as the SD (top); the product of the length (L) times the width (T) of the distribution in the Poincare plot (middle); and the RMSSD (bottom). The data for control pups are expressed as the mean shown as the long thin horizontal bar running across each panel, and the $95 \%$ confidence intervals shown as the short horizontal bars above and below the mean. Solid circles represent values at $\mathrm{P} 5 \mathrm{for}$ each "silenced" pup that ultimately survived. Open triangles represent values at $\mathrm{P} 5$ for each "silenced" pup that ultimately died. The symbols between the datasets for "survived" and "died" show a significant main effect of genotype. ${ }^{*} p=0.04 .{ }^{+} p=0.01$. The symbols above each dataset show post hoc significance ( $p=0.05$; Bonferroni).

Pet $1:$ Flpe-silenced mice, the SD and the RMSSD of the IBI were greater in silenced pups that died than controls $(p=$ 0.0125 and $p=0.0073$, respectively).

\section{Discussion}

\section{Strategy}

We hypothesized that brainstem abnormalities targeted to portions of the medullary 5-HT neuron population could impair the ability to autoresuscitate in early postnatal life (i.e., that normal serotonergic neurotransmission is essential to this vital process). We used Pet1::Flpe (Jensen et al., 2008) and Slc6a4::cre (Gong et al., 2007) driver lines coupled with the respective conditional tox (tetanus toxin light chain)-encoding R26 knock-in alleles (Kim et al., 2009) to selectively impair exocytic neurotransmitter release during development and then examined postnatally (P5, P8, and P12) the response to repeated anoxic stress such as to induce autoresuscitation.

\section{Main findings}

Significant findings include the following: (1) "Silencing" of portions of raphe neurons in triple transgenic Pet1::Flpe, hßactin::cre, RC::PFtox pups or double-transgenic Slc6a4::cre, RC::Ptox pups resulted in autoresuscitation failure at P5 and P8 but not P12. (2) There was no significant difference between the two experimental genotypes in this reduced ability to survive repeated anoxic stress. (3) In "silenced" pups of both experimental genotypes that died at P5, gasp latency was increased as were the times for recovery of $\mathrm{HR}$ and eupneic breathing, which were significantly correlated with the gasp latency. (4) In both experimental genotypes, analysis of baseline breathing variability at P5 could distinguish between "silenced" and control pups, but not predict future mortality. (5) In both experimental genotypes compared with controls, the pups were smaller and had slower HRs, slower breathing frequency, and larger tidal volumes, with variable effects on $\dot{V}_{E}$ and $\dot{V}_{\mathrm{O}_{2}}$. (6) In both models, there appear to be some driver-captured cells within the brainstem in which Tph2 expression is low or absent at P5 and P12, albeit more substantial in the Pet1::Flpe model. (7) Manipulated neurons shared between both experimental models were predominantly restricted to raphe serotonergic neurons.

Failed and delayed autoresuscitation in Slc6a4::cre- and Pet1::Flpe-silenced pups at a critical age

The drivers used to switch on tox expression in 5-HT neurons captured a portion of but not all medullary Tph2-immunopositive neurons at P5 and P12, approximately one-third of medullary 5 -HT neurons using Pet1:;Flpe; and approximately three-fourths using Slc6a4::cre, with the greatest overlap in manipulated cell populations in the dorsal raphe and the medullary region of the raphe magnus. These animal models, compared with others exhibiting greater serotonergic neuron deficiencies such as the 5,7-DHT-treated rat pups and the Lmx1b and Pet1-null mice (Hodges et al., 2009; Cummings et al., 2011a,b), may offer new pathophysiological insights into SIDS in which infants demonstrate a seemingly modest $\sim 26 \%$ deficiency in medullary 5-HT as measured from postmortem tissue punches (Duncan et al., 2010).

In both genetic models, the drivers captured some raphe neurons lacking in immunodetectable Tph2. In the case of the Pet1::Flpe driver, the affected neurons appear to be bona fide Pet1 expressors, suggesting the existence of a novel Pet $1^{+}$,

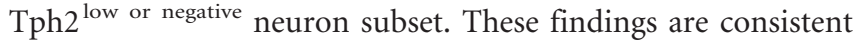
with our recent transcriptomic studies (Okaty et al., 2015) that uncovered a population of neurons defined as $\mathrm{Pet}{ }^{+}, \mathrm{Tph} 2^{\text {low }}$, Slc6a $4^{\text {low }}$; moreover, Pelosi et al. (2014) report a subset of Pet ${ }^{+}$ raphe neurons lacking detectable levels of $T p h 2$. While the silencing of these $\mathrm{Tph} 2^{\text {low or negative }}$ raphe neurons may contribute to the observed autoresuscitation defects, the simplest model points to the overlapping neuron subset captured by each of our two drivers given the similarity in defects across both models. This shared population is largely $\mathrm{Tph} 2^{+}$(given that the bulk of the Slc6a4::cre-captured cells are $\mathrm{Tph} 2^{+}$) and resident within the ra- 
phe. Further work will be required to understand the significance of these Pet1 ${ }^{+}, T p h 2^{\text {low }}$ neurons, and the developmental time patterns of features generally used to identify 5-HT neurons.

Dramatic phenotypes were observed in these mouse models, despite manipulating only a portion of the raphe neuron population. Mortality was increased significantly, and autoresuscitation prolonged during repeated exposure to anoxia at a critical period in postnatal development. The first week of postnatal development in rodents is a time of significant change in central neural pathways controlling cardiorespiratory function (WongRiley and Liu, 2005; Fong, 2010). From our results, P5-P8 appears to be a critical period in which a decrease in raphe neurotransmission can increase vulnerability during autoresuscitation from repeated exposure to anoxia. At P12, there was no difference in survival compared with controls. Our study did include added pups at P8 and P12 that consequently had fewer cumulative anoxic exposures, but the improved survival included both pups that had prior anoxic exposure and pups that did not.

The increased mortality in the pups with tox-perturbed neurons at P5 and P8 agrees with previous reports that repeated exposure to anoxia increases mortality in Pet1-null mouse pups at P8 but not P12 (Cummings et al., 2011a) and in 5,7-DHT-treated rat pups at P7-P10 (Cummings et al., 2011b). In these earlier studies, the serotonin deficiency in the medulla is more substantial. The critical period of increased vulnerability in the Pet1-null pups (P8) is similar to the critical period (P5-P8) in this study. In the 5,7-DHT-treated rat pups, more anoxic exposures (15) were required before autoresuscitation failed. Rats appear to be more tolerant of hypoxia than mice (Fong, 2010), which may reflect temporal differences in postnatal brain development between mouse and rat (Schneider and Norton, 1979). We suggest that even a moderate deficiency in raphe neuron function may substantially reduce the ability to autoresuscitate from anoxiainduced apnea in early postnatal life.

In both Pet1::Flpe- and Slc6a4::cre-silenced pups that eventually succumbed to anoxia, gasping was significantly delayed compared with surviving control pups. This finding is consistent with previous reports in other 5-HT-deficient animal models exposed to a single (Erickson and Sposato, 2009) or multiple bouts of anoxia (Cummings et al., 2011a,b). In previous studies and this study, gasping appeared normal once it began, which is in agreement with in situ studies in juvenile rats and mice that showed that 5-HT is not critical for gasp generation (Toppin et al., 2007; St-John and Leiter, 2008; St-John et al., 2009). Our results, however, suggest that 5-HT and/or raphe neuron function is critical for the timely initiation of gasping (Chen et al., 2013). Erickson and Sposato (2009) suggest that this prolonged gasp latency might reflect an altered hypoxic threshold for activation of gasping (Guntheroth and Kawabori, 1975; Lawson and Thach, 1977) in the pre-Bötzinger complex (Lieske et al., 2000; Paton et al., 2006), which contains hypoxic-sensitive neurons (Solomon, 2000; Neubauer and Sunderram, 2004). Serotonin is known to modulate the activity of pre-Bötzinger complex neurons (Hodges and Richerson, 2008; Ptak et al., 2009) and may enhance sensitivity of hypoxia-sensitive neurons within the pre-Bötzinger complex. When this excitatory stimulation from $5-\mathrm{HT}$ is reduced, as is likely in the "silenced" pups in this study, more severe hypoxia might be required to activate these neurons and initiate gasping.

Pet1::Flpe- and Slc6a4::cre-silenced pups, in addition to a prolonged gasp latency, took longer to recover normal HR and breathing frequency after the initiation of gasping than control pups that survived, similar to previously reported studies in neonatal 5-HT-deficient rodents (Erickson and Sposato, 2009; Cummings et al., 2011a,b). Cardiac resuscitation follows reoxygenation of the heart as a result of gasping, which in turn leads to respiratory resuscitation and resumption of normal body functions (Gershan et al., 1992). A delay in cardiac reoxygenation due to a delay in the initiation of gasping could account for the delay in the recovery of HR and eupneic breathing and by extension, contribute to autoresuscitation failure. In $\mathrm{BALB} / \mathrm{c}$ mice, when oxygen availability is delayed, autoresuscitation fails, suggesting that early gasps may be more effective in reoxygenating the heart and promoting successful autoresuscitation than later gasps (Gershan et al., 1990). In support of this conclusion, we found that the time to recover normal HR and breathing frequency was directly related to the gasp latency.

\section{Altered baseline breathing and HRV in Slc6a4::cre- and Pet1::Flpe-silenced pups}

We found that the variability of baseline breathing, but not HR, was consistently greater in "silenced" pups of both animal models compared with controls. The ability to identify groups at greater risk of autoresuscitation failure (i.e., are more vulnerable) is a significant finding that might be applicable to human infants.

In conclusion, we found that perturbations affecting even a modest portion of raphe neurons, including 5-HT neurons, can compromise autoresuscitation postnatally. Raphe neurons appear to act during early development to protect against lethal consequences of repeated exposure to anoxia, and P5-P8 is a critical period of increased vulnerability. The gasp latency is important in determining the speed and/or success of autoresuscitation and the timely initiation of gasping may be dependent on normal serotonergic signaling. Baseline measures of breathing variability identified the groups of animals at greater risk and may, therefore, be useful in identifying infants at an increased risk for SIDS. We conclude that the serotonergic deficiency, reflected as a decrease in tissue levels of 5-HT that has been documented in SIDS cases (Duncan et al., 2010), may alter respiratory and autonomic responses to severe hypoxia in early postnatal life and may potentially be causally related to the SIDS pathogenesis.

\section{References}

Adolph EF (1969) Regulations during survival without oxygen in infant mammals. Respir Physiol 7:356-368. CrossRef Medline

Brust RD, Corcoran AE, Richerson GB, Nattie E, Dymecki SM (2014) Functional and developmental identification of a molecular subtype of brain serotonergic neuron specialized to regulate breathing dynamics. Cell Rep 9:2152-2165. CrossRef Medline

Carrasco S, Gaitán MJ, González R, Yánez O (2001) Correlation among Poincare plot indexes and time and frequency domain measures of heart rate variability. J Med Eng Technol 25:240-248. CrossRef Medline

Chatow U, Davidson S, Reichman BL, Akselrod S (1995) Development and maturation of the autonomic nervous system in premature and full-term infants using spectral analysis of heart rate fluctuations. Pediatr Res 37: 294-302. CrossRef Medline

Chen J, Magnusson J, Karsenty G, Cummings KJ (2013) Time- and agedependent effects of serotonin on gasping and autoresuscitation in neonatal mice. J Appl Physiol 114:1668-1676. CrossRef Medline

Cummings KJ, Commons KG, Hewitt JC, Daubenspeck JA, Li A, Kinney HC, Nattie EE (2011a) Failed heart rate recovery at a critical age in 5-HTdeficient mice exposed to episodic anoxia: implications for SIDS. J Appl Physiol 111:825-833. CrossRef Medline

Cummings KJ, Hewitt JC, Li A, Daubenspeck JA, Nattie EE (2011b) Postnatal loss of brainstem serotonin neurones compromises the ability of neonatal rats to survive episodic severe hypoxia. J Physiol 589:5247-5256. CrossRef Medline

Deshpande P, Khurana A, Hansen P, Wilkins D, Thach BT (1999) Failure of autoresuscitation in weanling mice: significance of cardiac glycogen and heart rate regulation. J Appl Physiol 87:203-210. Medline 
Duncan JR, Paterson DS, Hoffman JM, Mokler DJ, Borenstein NS, Belliveau RA, Krous HF, Haas EA, Stanley C, Nattie EE, Trachtenberg FL, Kinney HC (2010) Brainstem serotonergic deficiency in sudden infant death syndrome. JAMA 303:430-437. CrossRef Medline

Erickson JT, Sposato BC (2009) Autoresuscitation responses to hypoxiainduced apnea are delayed in newborn 5-HT-deficient Pet- 1 homozygous mice. J Appl Physiol 106:1785-1792. CrossRef Medline

Fewell JE (2005) Protective responses of the newborn to hypoxia. Respir Physiol Neurobiol 149:243-255. CrossRef Medline

Fong AY (2010) Postnatal changes in the cardiorespiratory response and ability to autoresuscitate from hypoxic and hypothermic exposure in mammals. Respir Physiol Neurobiol 174:146-155. CrossRef Medline

Gershan WM, Jacobi MS, Thach BT (1990) Maturation of cardiorespiratory interactions in spontaneous recovery from hypoxic apnea (autoresuscitation). Pediatr Res 28:87-93. CrossRef Medline

Gershan WM, Jacobi MS, Thach BT (1992) Mechanisms underlying induced autoresuscitation failure in BALB/c and SWR mice. J Appl Physiol 72:677-685. Medline

Gong S, Doughty M, Harbaugh CR, Cummins A, Hatten ME, Heintz N, Gerfen CR (2007) Targeting cre recombinase to specific neuron populations with bacterial artificial chromosome constructs. J Neurosci 27: 9817-9823. CrossRef Medline

Guntheroth WG, Kawabori I (1975) Hypoxic apnea and gasping. J Clin Invest 56:1371-1377. CrossRef Medline

Harvey PJ, Li X, Li Y, Bennett DJ (2006) 5-HT2 receptor activation facilitates persistent sodium current and repetitive firing in spinal motoneurons of rats with and without spinal cord injury. J Neurophysiol 96: 1158-1170. CrossRef Medline

Hodges MR, Richerson GB (2008) Contributions of 5-HT neurons to respiratory control: neuromodulatory and trophic effects. Respir Physiol Neurobiol 164:222-232. CrossRef Medline

Hodges MR, Wehner M, Aungst J, Smith JC, Richerson GB (2009) Transgenic mice lacking serotonin neurons have severe apnea and high mortality during development. J Neurosci 29:10341-10349. CrossRef Medline

Jacobi MS, Gershan WM, Thach BT (1991) Mechanism of failure of recovery from hypoxic apnea by gasping in 17- to 23-day-old mice. J Appl Physiol 71:1098-1105. Medline

Jensen P, Farago AF, Awatramani RB, Scott MM, Deneris ES, Dymecki SM (2008) Redefining the serotonergic system by genetic lineage. Nat Neurosci 11:417-419. CrossRef Medline

Just A, Faulhaber J, Ehmke H (2000) Autonomic cardiovascular control in conscious mice. Am J Physiol Regul Integr Comp Physiol 279:R2214R2221. Medline

Kim JC, Cook MN, Carey MR, Shen C, Regehr WG, Dymecki SM (2009) Linking genetically defined neurons to behavior through a broadly applicable silencing allele. Neuron 63:305-315. CrossRef Medline

Kinney HC, Richerson GB, Dymecki SM, Darnall RA, Nattie EE (2009) The brainstem and serotonin in the sudden infant death syndrome. Ann Rev Pathol 4:517-550. CrossRef Medline

Lawson EE, Thach BT (1977) Respiratory patterns during progressive asphyxia in newborn rabbits. J Appl Physiol 43:468-474. Medline

Leiter JC, Böhm I (2007) Mechanisms of pathogenesis in the Sudden Infant Death Syndrome. Respir Physiol Neurobiol 159:127-138. CrossRef Medline

Lewandoski M, Meyers EN, Martin GR (1997) Analysis of Fgf8 gene function in vertebrate development. Cold Spring Harb Symp Quant Biol 62: 159-168. CrossRef Medline

Lieske SP, Thoby-Brisson M, Telgkamp P, Ramirez JM (2000) Reconfiguration of the neural network controlling multiple breathing patterns: eupnea, sighs and gasps. Nat Neurosci 3:600-607. CrossRef Medline

Madisen L, Zwingman TA, Sunkin SM, Oh SW, Zariwala HA, Gu H, Ng LL, Palmiter RD, Hawrylycz MJ, Jones AR, et al. (2010) A robust and highthroughput Cre reporting and characterization system for the whole mouse brain. Nat Neurosci 13:133-140. CrossRef Medline

Melton JE, Kadia SC, Yu QP, Neubauer JA, Edelman NH (1996) Respiratory and sympathetic activity during recovery from hypoxic depression and gasping in cats. J Appl Physiol 80:1940-1948. CrossRef Medline

Meny RG, Carroll JL, Carbone MT, Kelly DH (1994) Cardiorespiratory recordings from infants dying suddenly and unexpectedly at home. Pediatrics 93:44-49. Medline
Moody GB (1993) Spectral analysis of heart rate without resampling. Comut Cardiol 20:715-718.

Neubauer JA, Sunderram J (2004) Oxygen-sensing neurons in the central nervous system. J Appl Physiol 96:367-374. Medline

Okaty BW, Freret ME, Rood BD, Brust RD, Hennessy ML, deBairos D, Kim JC, Cook MN, Dymecki SM (2015) Multi-scale molecular deconstruction of the serotonin neuron system. Neuron 88:774-791. CrossRef Medline

Paton JFR, Abdala AP, Koizumi H, Smith JC, St-John WM (2006) Respiratory rhythm generation during gasping depends on persistent sodium current. Nat Neurosci 9:311-313. CrossRef Medline

Pelosi B, Migliarini S, Pacini G, Pratelli M, Pasqualetti M (2014) Generation of Pet1210-Cre transgenic mouse line reveals non-serotonergic expression domains of Pet1 both in CNS and periphery. PLoS One 9:e104318. CrossRef Medline

Peña F, Ramirez JM (2002) Endogenous activation of serotonin-2A receptors is required for respiratory rhythm generation in vitro. J Neurosci 22:11055-11064. Medline

Poets CF (2004) Apparent life-threatening events and sudden infant death on a monitor. Paediatr Respir Rev 5 [Suppl A]:S383-S386.

Poets CF, Meny RG, Chobanian MR, Bonofiglo RE (1999) Gasping and other cardiorespiratory patterns during sudden infant deaths. Pediatr Res 45:350-354. CrossRef Medline

Ptak K, Yamanishi T, Aungst J, Milescu LS, Zhang R, Richerson GB, Smith JC (2009) Raphe neurons stimulate respiratory circuit activity by multiple mechanisms via endogenously released serotonin and substance P. J Neurosci 29:3720-3737. CrossRef Medline

Ray RS, Corcoran AE, Brust RD, Kim JC, Richerson GB, Nattie EE, Dymecki SM (2011) Impaired respiratory and body temperature control upon acute serotonergic neuron inhibition. Science 333:637-642. CrossRef Medline

Saiki C, Ikeda M, Nishikawa T, Tanimoto T, Yoshida S, Matsumoto S (2001) The process of cardiorespiratory autoresuscitation in intact newborn rats. Can J Physiol Pharmacol 79:1036-1043. CrossRef Medline

Schiavo G, Matteoli M, Montecucco C (2000) Neurotoxins affecting neuroexocytosis. Physiol Rev 80:717-766. Medline

Schneider BF, Norton S (1979) Equivalent ages in rat, mouse and chick embryos. Teratology 19:273-278. CrossRef Medline

Solomon IC (2000) Excitation of phrenic and sympathetic output during acute hypoxia: contribution of medullary oxygen detectors. Respir Physiol 121:101-117. CrossRef Medline

Soriano P (1999) Generalized lacZ expression with the ROSA26 Cre reporter strain. Nat Genet 21:70-71. CrossRef Medline

Sridhar R, Thach BT, Kelly DH, Henslee JA (2003) Characterization of successful and failed autoresuscitation in human infants, including those dying of SIDS. Pediatr Pulmonol 36:113-122. CrossRef Medline

Stevens LH (1965) Sudden unexplained death in infancy: observations on a natural mechanism of the adoption of the face down position. Am J Dis Child 110:243-247. CrossRef Medline

St-John WM, Leiter JC (2008) Maintenance of gasping and restoration of eupnea after hypoxia is impaired following blockers of alphal-adrenergic receptors and serotonin 5-HT2 receptors. J Appl Physiol 104:665-673. CrossRef Medline

St-John WM, Li A, Leiter JC (2009) Genesis of gasping is independent of levels of serotonin in the Pet-1 knockout mouse. J Appl Physiol 107:679_ 685. CrossRef Medline

Thach BT (2008) Some aspects of clinical relevance in the maturation of respiratory control in infants. J Appl Physiol 104:1828-1834. CrossRef Medline

Toppin VA, Harris MB, Kober AM, Leiter JC, St-John WM (2007) Persistence of eupnea and gasping following blockade of both serotonin type 1 and 2 receptors in the in situ juvenile rat preparation. J Appl Physiol 103:220-227. CrossRef Medline

Tryba AK, Peña F, Ramirez JM (2006) Gasping activity in vitro: a rhythm dependent on 5-HT2A receptors. J Neurosci 26:2623-2634. CrossRef Medline

Wong-Riley MT, Liu Q (2005) Neurochemical development of brain stem nuclei involved in the control of respiration. Respir Physiol Neurobiol 149:83-98. CrossRef Medline 